\title{
Der Schulwettbewerb zur Entwicklungspolitik und seine Materialien - ein Schlaglicht
}

\begin{abstract}
Zusammenfassung
Die Globalisierung bringt wesentliche Veränderungen für jeden von uns mit sich. Sie sind Teil unserer Lebenswelt, der Umgang mit ihnen ist immer mehr Alltag - heute mehr denn je, Tendenz steigend. Unter dem Stichwort Bildung für nachhaltige Entwicklung (BNE) unterstützen die Angebote von Engagement Global Schülerinnen, Schüler und Lehrkräfte darin, sich diesen Veränderungen zu stellen. Ein Beispiel hierfür: Der Schulwettbewerb zur Entwicklungspolitik. Seit 2003 ruft dieser Schülerinnen und Schüler aller Altersstufen, Schulformen und -fächer dazu auf, sich mit der EINEN WELT auseinanderzusetzen. Ziel des Wettbewerbs ist es, Kinder und Jugendliche für nachhaltiges und verantwortliches Denken und Handeln in einer globalisierten Welt zu sensibilisieren und zu ermutigen, sich für diese zu engagieren. Um den Weg für Lehrkräfte wie Lernende für eine Auseinandersetzung mit Themen Globaler Entwicklung zu ebnen, bietet der Schulwettbewerb begleitend zu jedem Durchgang passende Unterrichtsmaterialien, die versuchen, der Breite und Pluralität der Bildungsansätze gerecht zu werden und gleichzeitig bedarfsgerechte, in der Praxis gut handhabbare und qualitativ hochwertige Unterrichtsvorschläge zur Verfügung zu stellen.
\end{abstract}

Schlüsselworte: BNE, Schulwettbewerb, Unterrichtsmaterial, Kompetenzerwerb

\footnotetext{
Abstract

Globalisation is bringing about major changes for each of us. They are part of our living environment, and dealing with them is more and more part of everyday life - today more than ever, and the trend is rising. Under the heading of Education for Sustainable Development (ESD), the services offered by Engagement Global support pupils and teachers in facing up to these changes. An example of this: The school competition on development policy. Since 2003, this competition has been calling on students of all ages, school types and subjects to engage with
}

ONE WORLD. The aim of the competition is to sensitize children and young people to sustainable and responsible thinking and acting in a globalized world and to encourage them to get involved. In order to pave the way for teachers and learners alike to deal with issues of global development, the school competition offers suitable teaching materials to accompany each round. These materials attempt to do justice to the breadth and plurality of educational approaches and at the same time contain teaching suggestions that are tailored to needs, easy to handle in practice and of high quality.

Keywords: ESD, school competition, learning material, acquisition of competencies

\section{Der Schulwettbewerb zur Entwicklungs- politik: EIN Weg zu BNE im Unterricht}

Ein Bewusstsein für einen nachhaltigen Umgang mit Themen Globaler Entwicklung zu schaffen, stellt eine gesamtgesellschaftliche Aufgabe dar. Der Lernort Schule bietet hierfür gute Voraussetzungen und kann maßgeblich dazu beitragen, dieser Aufgabe gerecht zu werden.

So geht es bei der Stärkung von BNE an Schulen vor allem darum, ein ganzheitliches Verständnis von nachhaltiger Entwicklung zu vermitteln und einen entsprechenden Kompetenzerwerb bei den Schülerinnen und Schülern anzuregen. Doch stellt die Behandlung von Themen Globaler Entwicklung nach wie vor für viele Lehrkräfte eine große Herausforderung dar und viele betrachten die Behandlung dieser Themen als zusätzliche Vermittlungsarbeit.

Unter dem Stichwort Bildung für nachhaltige Entwicklung (BNE) unterstützen die Angebote von Engagement Global Lehrkräfte daher darin, sich diesen Herausforderungen zu stellen. Ein Beispiel hierfür: Der Schulwettbewerb zur Entwicklungspolitik. Seit 2003 ruft dieser Schülerinnen und Schüler aller Altersstufen, Schulformen und -fächer dazu auf, sich mit der EINEN WELT auseinanderzusetzen. Ziel des Wettbe- 
werbs ist es, Kinder und Jugendliche für nachhaltiges und verantwortliches Denken und Handeln in einer globalisierten Welt zu sensibilisieren und zu ermutigen, sich für diese zu engagieren. Zahlreiche Anknüpfungspunkte für die Auseinandersetzung mit globalen Themen bietet der Wettbewerb alle zwei Jahre besonders durch sein rundenspezifisches Motto. In der aktuell neunten Runde wurden zu dem Thema „Meine, deine, unsere Zukunft?! Lokales Handeln - globales Mitbestimmen“ zahlreiche Beiträge eingereicht.

\section{Didaktische und inhaltliche Grundlage: Der Orientierungsrahmen für den Lernbereich Globale Entwicklung}

Die inhaltliche und didaktische Grundlage des Wettbewerbs bildet der Orientierungsrahmen für den Lernbereich Globale Entwicklung (Engagement Global 2016) (OR), dessen Kompetenzmodell den Bewertungskriterien und den didaktischen Begleitinstrumenten des Wettbewerbs zugrunde liegt. Für viele Fächer sind in vielen Bundesländern bereits BNE-relevante Inhalte in den Lehrplänen festgeschrieben (siehe Deutsche UNESCO-Kommission, o.J.). Dies vermittelt der Orientierungsrahmen für den Lernbereich Globale Entwicklung und bietet Hilfestellung, BNE direkt in den Lehrplanunterricht zu integrieren und den Unterricht qualitätsvoll zu gestalten.

Innerhalb von Fachkapiteln stellt der OR mögliche Unterrichtsthemen und allgemeine sowie fachbezogene Kompetenzen vor, die bis hin zu Leistungsanforderungen in Aufgabenbeispielen ausgearbeitet werden. Er dient aber nicht nur als ein Bezugsrahmen für die Entwicklung von Lehrplänen und schulischen Curricula, sondern macht ebenso deutlich, wie BNE an
Schulen verankert werden kann - nicht nur in den Unterrichtsfächern, sondern auch fächerübergreifend, von der Grundschule über die Sekundarstufe I bis zur beruflichen Bildung. Zugleich legt er die Grundlagen für eine Verankerung in der Sekundarstufe II.

\section{BNE und Kompetenzerwerb: Die Unter- richtsmaterialien des Schulwettbewerbs auf einen Blick}

Um den Weg für Lehrkräfte wie Lernende für eine Auseinandersetzung mit Themen Globaler Entwicklung zu ebnen und ihnen einen schnellen und einfachen Zugang zu ermöglichen, sind passende Unterrichtsmaterialien ein adäquates Hilfsmittel. Die Unterrichtsbeispiele des Schulwettbewerbs zur Entwicklungspolitik unterstützen Lehrkräfte somit bei der Unterrichtsgestaltung - eine der zentralen Aufgaben, die von Lehrenden ohnehin tagtäglich geleistet werden muss.

Die Herausforderung bei der Konzeption dieser Materialien besteht darin, der Breite und Pluralität der Bildungsansätze gerecht zu werden und gleichzeitig bedarfsgerechte, in der Praxis gut handhabbare und qualitativ hochwertige Unterrichtsvorschläge zu entwickeln. Denn auch für Lehrkräfte, die bisher wenig Erfahrung mit BNE haben, muss das Material leicht zugänglich sein - und unterschiedliche Fächer und Schulformen sowie die Interessen der Lernenden müssen mitgedacht werden.

Die Unterrichtsmaterialien des Schulwettbewerbs zur Entwicklungspolitik bieten daher Anknüpfungspunkte für eine Vielzahl von Schulfächern: Die beiden Materialhefte für die Grundschule (Klasse 1-6) und die Sekundarstufe (Klasse 7-13) enthalten jeweils mehrere Themendossiers, die für unter-

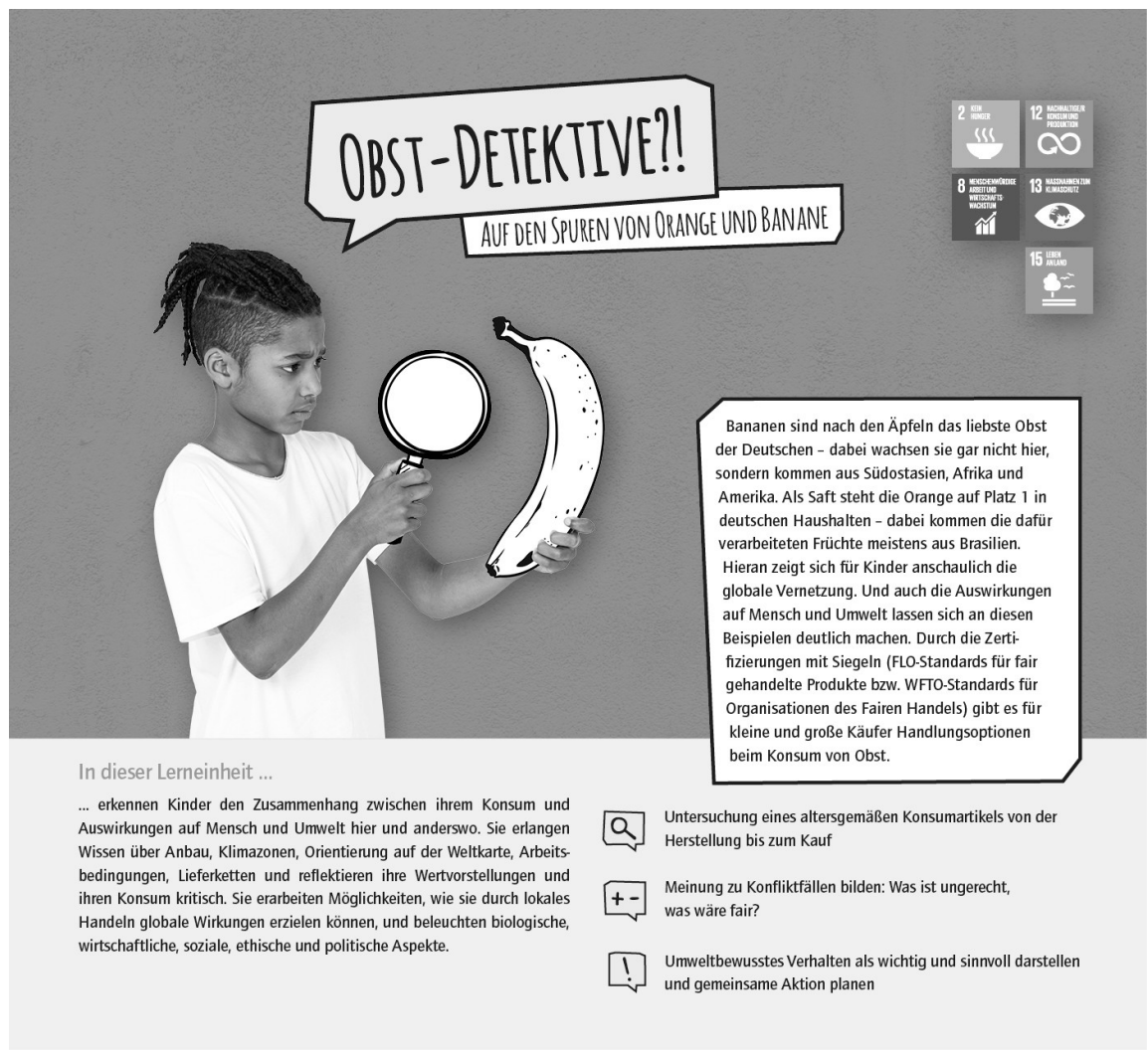

Abb. 1: Obst-Detektive, Lerneinheit aus dem aktuellen Unterrichtsmaterial zum Schulwettbewerb zur Entwicklungspolitik für die erste und zweite Klasse, Quelle: (c) Engagement Global, Fotograf: David Ertl 
schiedliche fachliche Schwerpunkte geeignet sind sowie eine Einführung, die die Grundsätze von BNE, den Orientierungsrahmen und sein Kompetenzmodell passend für den Unterricht in den jeweiligen Altersstufen zusammenfasst. Zudem wird die Agenda 2030 der Vereinten Nationen mit ihren 17 Zielen für nachhaltige Entwicklung als international gültiger Bezugsrahmen für den Themenkomplex globale Entwicklung erläutert.

Diese Punkte werden in den einzelnen Themendossiers wieder aufgegriffen und geben ihnen eine einheitliche Struktur, an der sich die Lehrkraft leicht orientieren kann: alle Dossiers sind klar entlang der drei Kompetenzbereiche Erkennen - Bewerten - Handeln gegliedert. Am Beginn jedes Dossiers befindet sich eine kurze Übersicht, mit deren Hilfe man auf einen Blick erfassen kann, welche Themen und Kompetenzen in dieser Einheit vermittelt werden und mit welchen der 17 Nachhaltigkeitsziele diese Themen verknüpft sind. So lässt sich unmittelbar entscheiden, ob die Lerneinheit passend für den eigenen Unterricht ist (vgl. Abb. 1).

Am Ende jedes Themendossiers ist ein QR-Code abgedruckt, der zu ergänzenden Arbeitsblättern sowie weiteren inhaltlich passenden Materialhinweisen auf der Webseite des Schulwettbewerbs führt, zum Beispiel zu Webseiten und Unterrichtsmaterialien anderer Organisationen, Videos, Presseartikeln u.v.m. Damit erleichtert das Material einen direkten, niedrigschwelligen Einstieg in BNE-Themen und hält den zusätzlichen Aufwand so gering wie möglich, um die Chancen auf die Umsetzung von BNE im Unterricht zu erhöhen. Um zugleich das Interesse der Schülerinnen und Schüler an diesem Bereich zu wecken, wurde besonders auf eine aktuelle und jugendaffine Themenauswahl geachtet, die sich im Alltag der Zielgruppe wiederfinden lässt.

\section{Alltagsthemen von Schülerinnen und Schülern im Fokus}

Jede Runde wird von einem thematischen Slogan begleitet, der sich stets am aktuellen Weltgeschehen orientiert und versucht, Themen aufzugreifen, die gerade im Fokus von Schülerinnen und Schülern stehen, um ihnen möglichst viele Anknüpfungspunkte für eine Auseinandersetzung mit globalen Themen zu bieten. So nutzten viele Lerngruppen in der Wettbewerbsrunde 2015/2016 das Thema „Umgang mit Vielfalt. Unterschiede verbinden - gemeinsam einzigartig!“ unter dem Eindruck der Ankunft zahlreicher Geflüchteter in Europa dazu, Beiträge zum Thema Flucht und Migration zu erarbeiten und einzureichen.

Ebenso wurde das Thema der Wettbewerbsrunde 2019/2020 „Meine, deine, unsere Zukunft?! Lokales Handeln - globales Mitbestimmen“ in 2020 von vielen Schülerinnen und Schülern mit der aktuellen Protestbewegung „Fridays for Future“ und dem Klimawandel in Verbindung gebracht. Hier kann BNE ansetzen, indem sie aktuelle (welt-)politische Ereignisse in einen Kontext setzt und aufzeigt, wie diese im Zusammenhang mit dem eigenen Leben und Handeln stehen. So haben wir dem Thema Klimawandel, das auch für immer mehr Jugendliche immer dringlicher wird, in den Unterrichtsmaterialien zur aktuellen Wettbewerbsrunde ein eigenes Themendossier gewidmet, in dem wissenschaftliche Fakten zur globa- len Erwärmung, die Folgen insbesondere für den Globalen Süden und innovative Lösungen für eine $\mathrm{CO}^{2}$-neutrale $\mathrm{Zu}$ kunft vorgestellt werden.

Aktuelle Themen sind aber nur ein möglicher Ausgangspunkt für BNE. Auch alltägliche Themen und Gegenstände abseits der großen politischen und medialen Bühne sind geeignet, die Gegenwart des Globalen im Alltag sichtbar zu machen - gerade für jüngere Schülerinnen und Schüler oder diejenigen, die sich (bisher) nicht besonders für Politik interessieren. Alltägliche Konsumprodukte wie Lebensmittel, Bekleidung oder Handys führen niedrigschwellig zu Fragen nach Produktionsbedingungen, Ressourcenverbrauch und möglichen Alternativen und lassen die Kinder und Jugendlichen erkennen, dass ihr lokales (Konsum-)Verhalten in einem globalen Zusammenhang steht.

Für die Grundschule eignen sich besonders Produkte, die zum (Aus-)Probieren einladen und keine zu komplizierte Lieferkette aufweisen. In einem der aktuellen Unterrichtsbeispiele für die Klassenstufen 1 und 2 wurde deshalb das Thema Obst mit den Beispielen Bananen und Orangen gewählt. Anhand dieser Beispiele können Kompetenzen wie die Orientierung auf der Weltkarte, die Untersuchung eines altersgemäßen Konsumartikels von der Herstellung bis zum Kauf, die Bewertung von Arbeitsbedingungen als fair oder unfair und das Planen gemeinsamer Aktionen eingeübt werden. Zudem wurden auch praktische Tätigkeiten wie gemeinsames Einkaufen und Kochen und spielerische Elemente wie z.B. ein Quiz in das Material eingebunden, um einen abwechslungsreichen und kindgerechten Unterricht zu ermöglichen.

In der Sekundarstufe kann die Auswahl der untersuchten Konsumartikel breiter und nach den individuellen Interessen der Schülerinnen und Schüler gestaltet werden. Je nach Altersstufe und Lernstand der Jugendlichen können zum Beispiel die Produktionswege von Textilien nachgezeichnet werden, es ist aber auch denkbar, auf einer abstrakteren Ebene die Problematik von Konsum, Ressourcenverbrauch und des ökologischen Fußabdrucks zu diskutieren. Das Themendossier „Konsum“ zur aktuellen Runde des Schulwettbewerbs bietet deshalb unterschiedliche Impulse zu Konsum und Lebensstil, Arbeitsbedingungen, Ressourcenverbrauch, Umweltbelastungen und nachhaltigem Konsum. Allen Impulsen ist gemeinsam, dass sie einen Kompetenzerwerb in den Bereichen Erkennen von global-lokalen Zusammenhängen, Bewertung von ökologischen und sozialen Folgen und Entwicklung von nachhaltigen Handlungsalternativen anregen.

Die unterschiedlichen Themen- und Unterrichtsvorschläge sind dabei immer lediglich als Orientierungshilfe und Impulsgeber für eine Beschäftigung mit globaler Nachhaltigkeit und zur Erstellung eines Wettbewerbsbeitrags zu verstehen. Denn der Schulwettbewerb zur Entwicklungspolitik zeichnet sich gerade durch eine große Offenheit gegenüber verschiedenen Themen und Formaten aus: Alles ist erlaubt, solange es dazu geeignet ist, bei den Schülerinnen und Schülern einen Kompetenzerwerb im Sinne von BNE anzuregen. Good-Practice-Beispiele aus den vergangenen Wettbewerbsrunden, die auf der Webseite und in Form einer Wanderausstellung verfügbar sind, bilden daher eine wichtige Ergänzung zu den Unterrichtsmaterialien und eine weitere Inspirationsquelle. 


\section{Die Wanderausstellung: Good Practice von Schulen für Schulen}

Runde für Runde erarbeiten Schülerinnen und Schüler nicht nur kreativ gestaltete und optisch beeindruckende Beiträge für die Teilnahme am Schulwettbewerb, sondern auch Beiträge, die eine inhaltlich tiefgreifende Auseinandersetzung mit globalen Themen im Unterricht erahnen lassen. Um auch andere von diesen beispielhaften Ergebnissen profitieren zu lassen, fließen diese in eine Wanderausstellung zum Schulwettbewerb ein. Diese besteht aus ausgewählten Beiträgen aus den vergangenen Durchgängen und wird jede Runde um neue Beiträge erweitert. Die Ausstellung wird interessierten Schulen und Bildungseinrichtungen für eine Dauer von zwei Wochen kostenfrei zur Verfügung gestellt, um diese bei der Auseinandersetzung mit Themen des Globalen Lernens im Unterricht, bei Projektwochen, Schulfesten oder anderen Veranstaltungen zu unterstützen. Vor dem Versand der Ausstellung findet immer ein Beratungsgespräch statt, um die Exponate den Bedarfen der Lerngruppe entsprechend zusammenzustellen: Nach Altersgruppe, Schulform, gewünschten Themen und Beitragsmedien.

Die Ausstellung basiert auf einem Peer-to-Peer-Ansatz, da die jeweilige Lerngruppe mit Produkten arbeitet, die andere Schülerinnen und Schüler erstellt haben. Im Idealfall können die Kinder und Jugendlichen so voneinander lernen. Ob ein Beitrag geeignet ist, einen solchen Multiplikationseffekt zu erzeugen und deshalb in die Ausstellung aufgenommen werden soll, wird sowohl im Bewertungsverfahren als auch bei der Auswahl der Beiträge für die Ausstellung berücksichtigt. Gleichzeitig spiegeln die ausgewählten Exponate die Vielfalt der Wettbewerbsbeiträge wieder, was Themen, Medien und Art der Umsetzung betrifft. So ermöglichen sie einen niedrigschwelligen Zugang zum Wettbewerb und können von der Lerngruppe als Inspiration für einen eigenen Wettbewerbsbeitrag genutzt werden.

Jede Ausleihe wird evaluiert, die Ergebnisse der Evaluation fließen in die weitere Aufbereitung der Ausstellung ein. Ein didaktisches Begleitheft zur Nutzung der Ausstellung ist in Konzeption, so dass Lehrkräften und Multiplikatoren perspektivisch ein methodisches Handwerkszeug zur Arbeit mit der Ausstellung mitangeboten wird. Zudem fließen Praxisbeispiele aus dem Wettbewerb auch in den Orientierungsrahmen und die Materialentwicklung ein und reichern ihn durch Impulse aus dem Schulalltag an. Beispielsweise wurde das Preisträger-

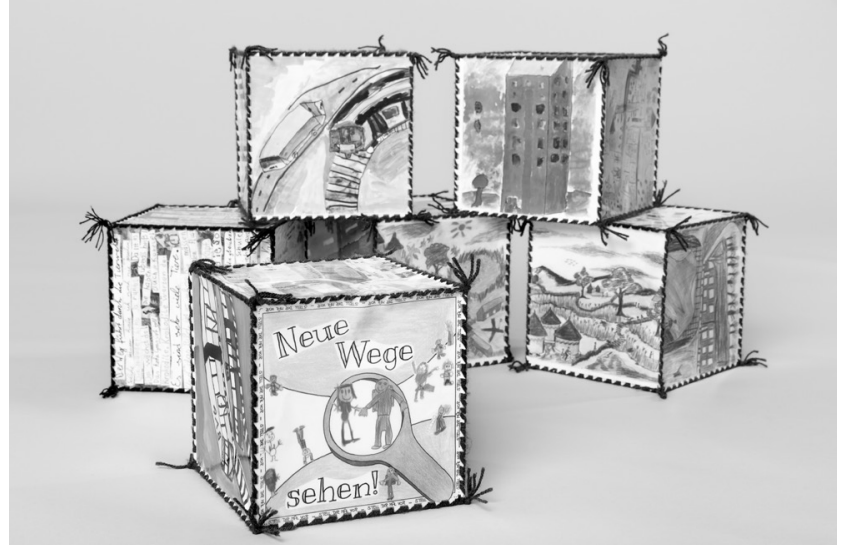

Abb. 2: Neue Wege sehen: Für diesen Wettbewerbsbeitrag tauschten sich Kinder aus Deutschland und Uganda per E-Mail über ihre Schulwege aus und verarbeiteten ihre Eindrücke in einem gemeinsamen Kunstprojekt, Quelle: (C) Engagement Global projekt „Neue Wege sehen“ aus dem Jahr 2012 für die überarbeitete Fassung des Orientierungsrahmens als Unterrichtsbeispiel aufbereitet.

\section{Expertise nutzen: Qualitätssicherung beim Schulwettbewerb}

Seit seinem Bestehen wird der Schulwettbewerb zur Entwicklungspolitik im Prozess der Materialentwicklung von Expertinnen und Experten unterschiedlicher Fachrichtungen begleitet. Es handelt sich hierbei um ein mehrstufiges Prozedere, bei dem Fachleute mit ihren unterschiedlichen Expertisen Aspekte wie die inhaltliche Ausrichtung des Materials, die didaktische Ausgestaltung und die Praxistauglichkeit in den Blick nehmen. In der Aufbereitung globaler Themen für den schulischen Kontext unterstützen erfahrene Autorinnen und Autoren bei der Erstellung der rundenspezifischen Unterrichtsmaterialien. Diese werden schließlich auf ihre praktische Anwendbarkeit hin im schulischen Kontext erprobt und im Rahmen der inhaltlichen Qualitätssicherungen durch die entsprechenden Fachreferate des BMZ auf ihre inhaltliche Richtigkeit geprüft. Um stets auch die Entwicklung pädagogischer Ansätze im Blick zu behalten, besteht besonders während des Materialentwicklungsprozesses in Vorbereitung einer jeden Wettbewerbsrunde ein ständiger Austausch mit pädagogischen Fachleuten aus dem Bereich BNE. Ein aktuelles Bespiel: In der Wettbewerbsrunde 2019/2020 wurde die methodisch-didaktische Beratung durch Mitarbeitende der Professur für „Inter- und transkulturelle Bildung und Sachunterricht “ der Leuphana Universität Lüneburg und des Seminars für Ausbildung und Fortbildung der Lehrkräfte Karlsruhe durchgeführt.

Die fachliche Beratung ging dabei von der Frage aus, wie sehr die bisher gewählten methodischen Ansätze tatsächlich in der Lage sind, Kompetenzen für eine globalisierte Welt gemäß den Zielen des Wettbewerbs zu vermitteln. Als Ergebnis der Beratung wurden einige strukturelle und methodische Neuerungen vorgenommen. Beispielsweise wurde im Material für die Grundschule der bisherige erzählende Ansatz mit Identifikationsfiguren durch einen produktorientierten Ansatz ersetzt, der noch unmittelbarer im Alltag der Kinder ansetzt. Beide Ansätze sind mögliche Ausgangspunkte für den Unterricht, die eine direkte Verbindung zur Lebenswelt der Kinder und zu den eigenen Handlungsmöglichkeiten zulässt. Wie in den vorhergegangenen Wettbewerbsrunden auch, werden die Kinder aufgefordert, ihre eigenen Ideen für eine gerechtere Welt zu erarbeiten. Bei der Materialerstellung für die Sekundarstufe wurde insbesondere auf eine weniger kleinteilige Struktur geachtet. Stattdessen wurden BNE-relevante Oberthemen - Entwicklungspolitik, Klima, Konsum, Kultur kompetenzorientiert aufbereitet und mit konkreten methodischen und thematischen Vorschlägen sowie begleitenden Arbeitsblättern versehen. Insgesamt wurden die Themenauswahl und der Aufbau des Materials noch stärker an der Lebenswelt der Schülerinnen und Schüler ausgerichtet, um Interesse an Themen der globalen Entwicklung zu wecken und einen Brückenschlag zu den eigenen Handlungsmöglichkeiten zu schaffen. Zugleich wurde darauf geachtet, das Material noch übersichtlicher und zugänglicher für die Lehrkraft zu gestalten, um eine direkte Anwendung im Unterricht zu ermöglichen. 


\section{Ausblick}

Um Lehrkräften die Möglichkeit zu geben, sich im Bereich Bildung für Nachhaltige Entwicklung weiterzubilden, bietet der Schulwettbewerb in regelmäßigen Abständen kostenfreie durch das BMZ finanzierte Fortbildungen für Lehrkräfte der Grundschule und Sekundarstufe in den Stufen „Anfänger“ und „Fortgeschrittene“ an. Dort erhalten die Teilnehmenden fachlichen Input von erfahrenen Referentinnen und Referenten aus dem Bereich der BNE, Informationen zum Wettbewerb und die Möglichkeit zum Austausch mit anderen Lehrkräften. In der Fortbildung für Grundschullehrkräfte im November 2019 wurde erstmalig ein neues Konzept erprobt, bei dem Lehrkräfte in verschiedenen Workshops die Möglichkeit hatten, mit den rundenspezifischen Unterrichtsmaterialien zu arbeiten. Die Teilnehmenden versetzten sich in die Rolle von Lernenden und probierten die Materialien selbst aus, anschließend tauschten sie sich zur Adaption ihres Materials für den eigenen Unterricht aus. Diese Art der Erprobung soll in Zukunft noch weiter ausgebaut werden und die Ergebnisse in die künftige Konzeption von Materialien einfließen. Ebenso soll bei der Materialerstellung in den nächsten Runden stärker auf die Expertise der NRO-Partner zurückgegriffen werden. Bereits in der aktuellen Runde wurden nicht nur eigene, sondern erstmals auch von den NRO-Partnern des Wettbewerbs selbst Bildungsmaterialien erstellt, die sich in den Themenkanon des aktuellen Rundenmottos einfügen. Die Idee dabei war zum einen, Lerngruppen von der Expertise unserer Partner in ihren Fachthemen wie zum Beispiel Wasser und Gesundheit, Kinderrechte, Inklusion und Geschlechtergerechtigkeit profitieren zu lassen. Zum anderen sollen Schulen und NROs Möglichkeiten aufgezeigt werden, zusammen zu arbeiten, um den Weg für Kooperationen zum Beispiel im Rahmen der Beitragserstellung für den Wettbewerb, aber auch darüber hinaus zu ebnen. Für die nächste Wettbewerbsrunde sind eine Neuentwicklung begleitender Materialien wie oben beschrieben, eine Verstetigung und Erweiterung des Fortbildungsangebots sowie die genannte didaktische Anreicherung der Ausstellung geplant. Zudem wird die
Online-Materialsammlung auf der Webseite des Wettbewerbs derzeit überarbeitet und soll in Zukunft durch eine optimierte Suche, verbesserte Filtermöglichkeiten und kurze Beschreibungstexte zu den Materialien Nutzerinnen und Nutzern eine bessere Orientierung bieten. Alle didaktischen Instrumente des Wettbewerbs werden dabei beständig auf ihre Nutzerfreundlichkeit und Wirksamkeit hin geprüft, damit Lehrkräfte weiterhin mit qualitativ hochwertigen Angeboten bei der Umsetzung von BNE in ihrem Unterricht unterstützt werden können.

\section{Literatur}

Engagement Global (Hrsg.) (2016). Orientierungsrahmen für den Lernbereich Globale Entwicklung im Rabmen einer Bildung für Nachhaltige Entwicklung (2. aktualisierte und erweiterte Auflage) Berlin: Cornelsen. Zugriff am: 01.06.2020 http://ww w.globaleslernen.de/sites/default/files/files/link-elements/orientierungsrahmen_ fuer_den_lernbereich_globale_entwicklung_barrierefrei.pdf

Engagement Global (Hrsg.) (2019). Meine, deine, unsere Zukunft?! Lokales Handeln Globales Mitbestimmen. Unterrichtsmaterialien für die Klassen 1-6. Bonn: Cornelsen Zugriff am: 01.06.2020 https://www.eineweltfueralle.de/fileadmin/user_upload/ unterrichten/material_klassen_1_bis_6/eg_swb_2019_umat-abb_klassen_1-6.pdf Engagement Global (Hrsg.) (2019). Meine, deine, unsere Zukunft?! Lokales Handeln Globales Mitbestimmen. Unterrichtsmaterialien für die Klassen 7-13. Bonn: Cornelsen. Zugriff am 01.06.2020 https://www.eineweltfueralle.de/fileadmin/user_upload/unterrichten/material_klassen_7_bis_13/eg_swb_2019_umat-abb_klassen_7-13.pdf

Deutsche UNESCO-Kommission e.V. (o. J.). BNE-Portal. Bundesländer. Zugriff am 01.06.2020 https://www.bne-portal.de/de/bundesweit/bundesl\%C3\%A4nder

\section{Wiebke Schwinger}

Seit 2016 Projektkoordinatorin im Schulwettbewerb zur Entwicklungspolitik mit dem Arbeitsschwerpunkt Didaktik. Zuvor u. a. Mitarbeit in einem Forschungsprojekt zu politischen Engagement-Biographien Jugendlicher und junger Erwachsener.

Allgemeine Wettbewerbsdaten: Der „Schulwettbewerb zur Entwicklungspolitik“ hat zum Ziel, Kinder und Jugendliche für nachhaltiges und verantwortliches Denken und Handeln in der globalisierten Welt zu sensibilisieren. Seit 2003 findet er alle zwei Jahre statt, aktuell befindet sich die Runde 2019/2020 in der Bewertungsphase. Beiträge zur nächsten Wettbewerbsrunde können voraussichtlich ab Herbst 2021 eingereicht werden. Der Wettbewerb richtet sich an Schülerinnen und Schüler aller Altersstufen und Schulformen. Die Teilnahme erfolgt in vier Alterskategorien, zudem können sich Schulen mit einer besonderen Profilbildung im Bereich Bildung für nachhaltige Entwicklung um den Schulpreis bewerben. Eingereicht werden können Beiträge aus allen Fächern und in jeder erdenklichen Form (z. B. Dokumentationen, Filme, Kunstobjekte, gemeinschaftlich erstellte Facharbeiten). Der Wettbewerb wird vom Bundesministerium für wirtschaftliche Zusammenarbeit und Entwicklung beauftragt und finanziert und seit 2012 von Engagement Global durchgeführt. Der Bundespräsident ist Schirmherr des Wettbewerbs. Begleitend zum Schulwettbewerb wird der Song Contest „Dein Song für EINE WELT!“ durchgeführt, der Kinder und Jugendliche im Alter von 10 bis 25 Jahren dazu aufruft, sich musikalisch mit Themen globaler Entwicklung auseinanderzusetzen. Weitere Informationen zu den Wettbewerben gibt es unter www.eineweltfueralle.de und www.eineweltsong.de.

Partner der Wettbewerbe: Der Schulwettbewerb zur Entwicklungspolitik und der Song Contest werden in jeder Runde von Fachpartnern sowie Partnern aus den Bereichen Medien, Wirtschaft und Zivilgesellschaft unterstützt. In der Wettbewerbsrunde 2019/2020 sind dies: der Cornelsen Verlag, Christoffel-Blindenmission Deutschland e.V., Deutsches Medikamentenhilfswerk action medeor e.V., Kindernothilfe e.V., Plan International e.V. und SPIESSER - Die Jugendzeitschrift. Der Schulwettbewerb wird außerdem vom Grundschulverband e.V. als Fachpartner sowie von Zeit für die Schule als Medienpartner unterstützt. Der Verband deutscher Musikschulen e.V. ist Fachpartner des Song Contests. Backspin, BigFM und die Blogrebellen begleiten den Song Contest als Medienpartner. Darüber hinaus unterstützen prominente Patinnen und Paten den Song Contest.

Abb. 3: Infokasten mit den Rahmendaten zum Wettbewerb, Quelle: eigene Darstellung 